\title{
De Novo Synthesis of 2-Substituted Syn-1,3-Diols Via an Iterative Asymmetric Hydration Strategy
}

\author{
Md. Moinuddin Ahmed, Matthew S. Mortensen, and George A. O'Doherty \\ Department of Chemistry, West Virginia University, Morgantown, WV 26506
}

\section{Abstract}<smiles>[R]C=C(C)C=CC(=O)O[R20]OC=C=COC(=O)C=C[C@@H](C)C([R])O</smiles>

\begin{abstract}
The enantioselective syntheses of several protected 4-substituted syn-3,5-dihydroxy carboxylic esters have been achieved from the corresponding achiral $(E, E)$ or $(E, Z)-1,3$-dienoates. The route relies upon an enantio- and regioselective Sharpless dihydroxylation and a palladium-catalyzed reduction to form $\gamma$-substituted $\delta$-hydroxy-1-enoates. The resulting $\delta$-hydroxy-1-enoates are subsequently converted into benzylidene protected 4-substituted syn-3,5-dihydroxy carboxylic esters in one step. The benzylidene protected 3,5-dihydroxy carboxylic esters are produced in good overall yields ( $20 \%$ to $54 \%$ ) and high enantiomeric excess (73\%ee to $97 \%$ ee).
\end{abstract}

As part of our continuing program focused on the de novo asymmetric synthesis of polyketide based natural products, ${ }^{1}$ we developed a sequential hydration approach (enantioselective hydration 1 to 2 and a diastereoselective hydration 2 to 3 ) that converts achiral conjugated dienoates into enantiomerically enriched benzylidine protected syn-3,5-dihydroxyesters. ${ }^{2}$ The transformation relies upon a Sharpless asymmetric dihydroxylation followed by a Pd- $\pi$-allyl catalyzed allylic reduction to control both the regio-and enantioselectivity of the first hydration, and an Evans hemiacetal addition to achieve diastereoselectivity in the second hydration. ${ }^{3}$

With the successful application of this approach to various 1,3-polyol natural products, we targeted the structurally more complex polyene-polyol macrolides (e.g., mycoticin $\mathrm{A}^{4 \text {, Figure }}$ 1). Thus we required a strategy that would address two structural motifs of mycoticin $A$. That is to say, we required access to both 2-methyl-1,3-diol ( $C$-11 to $C$-17), 3,4 and $\delta$-hydroxy- $\gamma$ methyl enoate $(C-27$ to $C$-31) subunits. Other approaches to $\delta$-hydroxy- $\gamma$-methyl enoate synthons usually involve crotylation/metathesis, aldol/Wittig, or vinylogous aldol sequences; a few other more diverse strategies have been employed in recent years, as well. ${ }^{5}$

Unfortunately, our initial studies on a new catalytic asymmetric approach using various carbon nucleophiles to install an alkyl group at $C$-4 was met with little success (i.e., replacing the palladium hydride with a palladium alkyl in the Pd- $\pi$-allyl intermediate 5).

Alternatively, we envisioned that these two structural features could be prepared from $C-4$ substituted achiral dienoates by an iterative hydration approach (Scheme 1). This, of course,

George.ODoherty@mail.wvu.edu.

Supporting Information Available: Complete experimental procedures and spectral data for all new compounds can be found in the Supporting Information. This material is available free of charge via the Internet at http://pubs.acs.org. 
required that the initial asymmetric hydration reaction be stereospecific, which was demonstrated by substituting $\mathrm{DCO}_{2} \mathrm{H}$ for $\mathrm{HCO}_{2} \mathrm{H}$ (Scheme $1, \mathrm{R}_{3}=\mathrm{H}$; see supporting information). Then, we embarked on an effort to expand the asymmetric hydration methodology to include substituted dienoates (1) for the preparation of the 4-methyl-5hydroxyenoates (2) and the benzylidine protected 4-methyl-3,5-dihydroxy esters (3) via the substitution of cyclic carbonates $\mathbf{4}$ and Pd- $\pi$-allyl intermediates 5 . $^{6}$ Herein, we describe the successful development of a de novo asymmetric synthesis of these two structural motifs ( 2 and $\mathbf{3}$ ) from simple achiral dienoates (1).

While we were initially concerned about the problems associated with enantio- and regioselectivity in both the osmium and palladium steps, the initial dienoates $(\mathbf{6 a} / \mathbf{b})$ we chose to study proved to be very promising (Scheme 2) ${ }^{7}$ Thus, exposure of dienoates $6 \mathbf{6}$ and $\mathbf{6 b}$ to Sharpless dihydroxylation conditions proceeded uneventfully (Table 1), providing diols $\mathbf{7 a}$ and 7b in good yields and excellent enantioselectivity. Similarly, the resulting diols were diastereoselectively converted to homo-allylic alcohols $\mathbf{8 a}$ and $\mathbf{8 b}$ by conversion to a cyclic carbonate and reduction with $\mathrm{Et}_{3} \mathrm{~N} \cdot \mathrm{HCO}_{2} \mathrm{H}$ (Table 2). ${ }^{8}$ Finally, both homo allylic alcohols $8 \mathbf{a}$ and $8 \mathbf{b}$ were readily converted into the benzylidine protected syn-3,5-dihydroxyester $9 \mathbf{a}$ and $\mathbf{9 b}$ by exposure to the Evans conditions ( $\mathrm{PhCHO}$, cat. $\mathrm{KO}$ - $\mathrm{Bu}$, Table 3 ).

Unfortunately, when we investigated the scope of this reaction sequence we uncovered complications with the dihydroxylation step (Table 1). The simplest dienoate substrate (Table 1 , entry d, R $=\mathrm{Me})^{9}$ underwent dihydroxylation using $(\mathrm{DHQ})_{2} \mathrm{PHAL}$ and (DHQD) $)_{2} \mathrm{PHAL}$ with excellent enantio- and regioselectivity; however, the regioselectivities were diminished for branched-alkyl and aryl substituents (Table 1, entries a-c). ${ }^{10}$ For instance, when dienoate $10 \mathrm{c}(\mathrm{R}=\mathrm{Ph})$ was dihydroxylated with the PHAL-linked dimeric ligands the $\alpha, \beta$-olefin $12 \mathrm{c}$ was preferentially formed (2.5:1). To our delight, switching to a "first-generation" dihydroxylation ligand, DHQ-4-Me-2-Quinolyl ether (DHQ-MEQ), eliminated this problem and gave the desired diol with excellent selectivity in all three cases (entries a-c, Table 1) with greatly improved regio- (> 16:1) and enantioselectivity (73-90\%ee) for the diols 11a-c.

The palladium catalyzed reduction proved to be very tolerant to a variety of functionalities, giving excellent yields and selectivities in all cases. As with the diols $\mathbf{7 a} / \mathbf{b}$, the diastereomerically pure diols $\mathbf{1 1 a - e}$ were converted into the corresponding cyclic carbonates 13a-e (Table 2) in excellent yields using triphosgene and pyridine in $\mathrm{CH}_{2} \mathrm{Cl}_{2}$. We next examined the Pd-catalyzed reduction of the $(E, E)$-allylic carbonates 13a-e. After some experimentation it was found that the optimal conditions were $1 \% \mathrm{Pd}_{2}(\mathrm{dba})_{3}{ }^{\circ} \mathrm{CHCl}_{3} / \mathrm{PPh}_{3}$ in THF with 5 equiv. of $\mathrm{Et}_{3} \mathrm{~N} \cdot \mathrm{HCO}_{2} \mathrm{H} .{ }^{11}$ In all case the carbonates were cleanly converted into homoallylic alcohols in excellent yields (>95\%). It is worth noting that we have been able to use this procedure for the preparation of multigram quantities of $14 \mathrm{~b}$ (i.e., several $10 \mathrm{~g}$ batches).

To demonstrate both the synthetic utility of this oxidation/reduction sequence as well as to assign the stereochemistry of the asymmetric hydration reaction the homoallylic alcohols 14ad were converted into the 1,3-syn diols 15a-d. Thus exposure of alcohols 14a-d to the Evans 1,3-syn diol protocol provided the benzylidine protected syn-3,5-dihydroxyesters 15a-d. With the exception of the phenyl substituted substrate $\mathbf{1 4} \mathbf{c}$ the benzylidine acetals were formed in good yields (57 to $75 \%$, Table 3 ). ${ }^{12}$

We next set out to test the stereospecificity of the overall transformation (6/10 to $9 / \mathbf{1 5})$. To do so we chose the (Z,E)-methyl substituted dienoate $\mathbf{1 6}$ (c.f., Table 4 and Scheme 3). Once again the initial dihydroxylation proved to be problematic. While no regioisomers were detected, the enantioselectivities were unsatisfactory using the PHAL-linked dimeric ligands (Table 4 entries 1-2). 
We again turned to the DHQ-MEQ ligand but this time we were met with lower ee (entry 3, Table 4). A screening of commercially-available AD ligands was conducted in which the optimum ligand was determined to be (DHQD) ${ }_{2} \mathrm{PYR}$ (entry 4, Table 4). ${ }^{13}$ In an effort to further increase the enantioselectivity, a Shi epoxidation ${ }^{14}$ was attempted on $\mathbf{1 6}$ (Scheme 3). Indeed, epoxide 19 was formed in greater enantioexcess and was subjected to identical Pd-reduction conditions as the carbonate $\mathbf{1 8}$. Both $\mathbf{1 8}$ and $\mathbf{1 9}$ behaved similarly in the reaction giving excellent $\mathrm{dr}$ with the epoxide opening having higher yield (98\% vs $86 \%$ ). Finally, the conversion of $\mathbf{2 0}$ to the anti-methyl diastereomer $\mathbf{2 1}$ occurred in $80 \%$ yield and diastereoselectivity (> 95:5) via the Evans protocol. ${ }^{15}$

In summary we have demonstrated the utility of our asymmetric bis-hydration methodology for the stereospecific conversion of both $(E, E)$ - and $(E, Z)$-dienoates into either $C$-4 diastereomer of benzylidine protected syn-3,5-dihydroxy esters $\left(\mathbf{9}, \mathbf{1 5}\right.$ and 21) ${ }^{9}$ Key to this development was the control of regioselectivity in both the osmium-catalyzed asymmetric dihydroxylation and palladium-catalyzed reduction reactions. Further development to improve the enantioselectivity of the oxidation of the $(E, Z)$-dienoates and its application toward natural product synthesis is ongoing.

\section{Experimental Section 16}

\section{General Procedure for dihydroxylations}

Into a round bottom flask containing $\mathrm{K}_{3} \mathrm{Fe}(\mathrm{CN})_{6}$ ( 3 equiv.), $\mathrm{K}_{2} \mathrm{CO}_{3}$ (3 equiv.), $\mathrm{MeSO}_{2} \mathrm{NH}_{2}$ (3 equiv.) and (DHQ) $)_{2}$-PHAL ( $\left.5 \mathrm{~mol} \%\right)$ was added $t-\mathrm{BuOH}$ and water $(1: 1,0.2 \mathrm{M})$. The mixture was stirred at $0^{\circ} \mathrm{C}$ for 5 minutes and then to this solution was added $\mathrm{OsO}_{4}(1 \mathrm{~mol} \%)$ immediately followed by addition of dienoate. The reaction was stirred vigorously at $0^{\circ} \mathrm{C}$ for $2-18 \mathrm{~h}$. Ethyl acetate was added to the reaction mixture followed by quenching with solid sodium sulfite. The layers were separated and the aqueous layer was extracted with EtOAc. The combined organic layers were washed with brine and then dried over $\mathrm{Na}_{2} \mathrm{SO}_{4}$. After concentration the crude mixture was purified by silica gel column chromatography.

(+)-(E,4S,5S)-Ethyl 7-(tert-butyldimethylsilyloxy)-4,5-dihydroxy-4-methylhept-2enoate (7a)-After flash column chromatography (30\% EtOAc/hexanes) the reaction yielded $380 \mathrm{mg}(68 \%)$ of diol as a clear, colorless oil. $R_{f}=0.10\left(4: 1\right.$ hexanes:EtOAc), $[\alpha]_{\mathrm{D}}{ }^{24}+5.2(c$ 0.61, $\mathrm{CH}_{2} \mathrm{Cl}_{2}$ ); IR (neat, cm ${ }^{-1}$ ) 3459(br), 2932, 2859, 1713, 1658, 1469, 1369, 1257, 1183, $1089,987,941,836,778,728 ;{ }^{1} \mathrm{H}$ NMR $\left(\mathrm{CDCl}_{3}, 600 \mathrm{MHz}\right) \delta 6.99(\mathrm{~d}, J=15.6 \mathrm{~Hz}, 1 \mathrm{H}), 6.11$ $(\mathrm{d}, J=15.6 \mathrm{~Hz}, 1 \mathrm{H}), 4.17(\mathrm{q}, J=7.2 \mathrm{~Hz}, 2 \mathrm{H}), 3.90(\mathrm{~m}, 2 \mathrm{H}), 3.74(\mathrm{dd}, J=6,6 \mathrm{~Hz}, 1 \mathrm{H}), 1.99$ (brs, $2 \mathrm{H}), 1.71(\mathrm{~m}, 2 \mathrm{H}), 1.27(\mathrm{t}, J=7.2 \mathrm{~Hz}, 3 \mathrm{H}), 1.25(\mathrm{~s}, 3 \mathrm{H}), 0.88(\mathrm{~s}, 9 \mathrm{H}), 0.07(\mathrm{~s}, 6 \mathrm{H}) ;{ }^{13} \mathrm{C}$ $\mathrm{NMR}\left(\mathrm{CDCl}_{3}, 150 \mathrm{MHz}\right) \delta 166.6,152.3,120.2,77.2$, 74.6, 62.5, 60.3, 32.2, 25.7 (3C), 22.8, 18.0, 14.1, -5.6 (2C); HRMS (ESI) calcd for $\left[\mathrm{C}_{16} \mathrm{H}_{32} \mathrm{O}_{5} \mathrm{Si}+\mathrm{Na}\right]^{+}: 355.1911$, Found: 355.1909 .

(+)-(E,4S,5S)-Ethyl 7-(benzyloxy)-4,5-dihydroxy-4-methylhept-2-enoate (7b)After purification by flash column chromatography (50\% EtOAc/hexanes) the diol was obtained in $71 \%$ yield as a clear, colorless oil. $R_{f}=0.11\left(4: 1\right.$ hexanes:EtOAc), $[\alpha]_{\mathrm{D}}{ }^{24}+5.2(c$ 2.0, $\mathrm{CH}_{2} \mathrm{Cl}_{2}$ ); IR (neat, $\mathrm{cm}^{-1}$ ) 3461(br), 2931, 2860, 1715, 1453, 1367, 1282, 1186, 1095, 1031, 987, 698; ${ }^{1} \mathrm{H}$ NMR $\left(\mathrm{CDCl}_{3}, 600 \mathrm{MHz}\right) \delta 7.35(\mathrm{~m}, 3 \mathrm{H}), 7.30(\mathrm{~m}, 2 \mathrm{H}), 6.99(\mathrm{~d}, J=15.6 \mathrm{~Hz}$, $1 \mathrm{H}), 6.11(\mathrm{~d}, J=15.6 \mathrm{~Hz}, 1 \mathrm{H}), 4.52(\mathrm{~s}, 2 \mathrm{H}), 4.18(\mathrm{q}, J=7.2 \mathrm{~Hz}, 2 \mathrm{H}), 3.75(\mathrm{~m}, 2 \mathrm{H}), 3.68(\mathrm{~m}$, $1 \mathrm{H}), 3.51(\mathrm{~s}, 1 \mathrm{H}), 2.71(\mathrm{~s}, 1 \mathrm{H}), 1.81(\mathrm{~m}, 2 \mathrm{H}), 1.28(\mathrm{t}, J=7.2 \mathrm{~Hz}, 3 \mathrm{H}), 1.27(\mathrm{~s}, 3 \mathrm{H}) ;{ }^{13} \mathrm{C}$ NMR $\left(\mathrm{CDCl}_{3}, 150 \mathrm{MHz}\right) \delta 166.5,152.0,138.1,128.5(2 \mathrm{C}), 127.9,127.7$ (2C), 120.3, 76.7, 74.6, 73.5, 69.2, 60.4, 30.2, 22.8, 14.2; HRMS (ESI) calcd for $\left[\mathrm{C}_{17} \mathrm{H}_{24} \mathrm{O}_{5}+\mathrm{Na}\right]^{+}: 331.1515$, Found: 331.1516.

(-)-(E,4S,5S)-Ethyl 4,5-dihydroxy-4,7-dimethyloct-2-enoate (11a)-After purification by flash column chromatography (50\% EtOAc/hexanes) the reaction yielded 44 
$\mathrm{mg}(75 \%)$ of diol as a clear oil with no detectable regioisomer. $R_{f}=0.15$ (4:1 hexanes:EtOAc), $[\alpha]_{\mathrm{D}}{ }^{24}-21.7\left(c 1.0, \mathrm{CH}_{2} \mathrm{Cl}_{2}\right)$; IR (neat, $\left.\mathrm{cm}^{-1}\right) 3460,2957,2870,1701,1656,1466,1368,1307$, $1282,1189,1034,988,869,766,743,652 ;{ }^{1} \mathrm{H} \mathrm{NMR}\left(\mathrm{CDCl}_{3}, 600 \mathrm{MHz}\right) \delta 6.95(\mathrm{~d}, J=15.6$ $\mathrm{Hz}, 1 \mathrm{H}), 6.10(\mathrm{~d}, J=15.6 \mathrm{~Hz}, 1 \mathrm{H}), 4.19(\mathrm{q}, J=7.2 \mathrm{~Hz}, 2 \mathrm{H}), 3.56(\mathrm{ddd}, J=10.8,4.2,2.4 \mathrm{~Hz}$, $1 \mathrm{H}), 2.53$ (brs, $1 \mathrm{H}), 2.31$ (brs, $1 \mathrm{H}), 1.80(\mathrm{~m}, 1 \mathrm{H}), 1.38$ (ddd, $J=13.8,10.8,3.6 \mathrm{~Hz}, 1 \mathrm{H}), 1.28$ $(\mathrm{t}, J=7.2 \mathrm{~Hz}, 3 \mathrm{H}), 1.26(\mathrm{~s}, 3 \mathrm{H}), 1.20(\mathrm{ddd}, J=13.8,10.2,4.2 \mathrm{~Hz}, 1 \mathrm{H}), 0.94(\mathrm{~d}, J=6.6 \mathrm{~Hz}$, $3 \mathrm{H}), 0.88(\mathrm{~d}, J=6.6 \mathrm{~Hz}, 3 \mathrm{H}) ;{ }^{13} \mathrm{C} \mathrm{NMR}\left(\mathrm{CDCl}_{3}, 150 \mathrm{MHz}\right) \delta 166.6,151.9,120.6,75.4,74.7$, $60.5,39.7,24.6,23.8,21.8,21.2,14.1$; HRMS (ESI) calcd for $\left[\mathrm{C}_{12} \mathrm{H}_{22} \mathrm{O}_{4}+\mathrm{Na}\right]^{+}: 253.1410$, Found: 253.1402 .

(-)-(E,4S,5S)-Ethyl 4,5-dihydroxy-4,6-dimethylhept-2-enoate (11b)-After purification by silica gel column chromatography (30\% EtOAc/hexanes) the reaction yielded $72 \mathrm{mg}(60 \%)$ of diols as a 16:1 mixture of regioisomers. $R_{f}=0.11$ (4:1 hexanes:EtOAc), $[\alpha]_{\mathrm{D}}^{24}-3.0\left(c 0.5, \mathrm{CH}_{2} \mathrm{Cl}_{2}\right)$; IR (neat, $\left.\mathrm{cm}^{-1}\right) 3448,2962,2874,1698,1655,1467,1368,1303$, $1279,1179,1096,1031,984,869,725,679 ;{ }^{1} \mathrm{H} \mathrm{NMR}\left(\mathrm{CDCl}_{3}, 600 \mathrm{MHz}\right) \delta 6.89(\mathrm{~d}, J=15.6$ $\mathrm{Hz}, 1 \mathrm{H}), 5.99$ (d, $J=15.6 \mathrm{~Hz}, 1 \mathrm{H}), 4.10(\mathrm{q}, J=7.2 \mathrm{~Hz}, 2 \mathrm{H}), 3.27(\mathrm{dd}, J=4.8,3.6 \mathrm{~Hz}, 1 \mathrm{H})$, $2.32(\mathrm{~s}, 1 \mathrm{H}), 1.95(\mathrm{~d}, J=4.8 \mathrm{~Hz}, 1 \mathrm{H}), 1.86(\mathrm{~m}, 1 \mathrm{H}), 1.22(\mathrm{~s}, 3 \mathrm{H}), 1.19(\mathrm{t}, J=7.2 \mathrm{~Hz}, 3 \mathrm{H}), 0.90$ $(\mathrm{d}, J=6.6 \mathrm{~Hz}, 3 \mathrm{H}), 0.89(\mathrm{~d}, J=6.6 \mathrm{~Hz}, 3 \mathrm{H}) ;{ }^{13} \mathrm{C} \mathrm{NMR}\left(\mathrm{CDCl}_{3}, 150 \mathrm{MHz}\right) \delta 166.8,153.4$, $120.2,80.1,75.6,60.7,29.0,23.1,22.0,16.6,14.4$; HRMS (ESI) calcd for $\left[\mathrm{C}_{11} \mathrm{H}_{20} \mathrm{O}_{4}+\right.$ $\mathrm{Na}]^{+}:$239.1253, Found: 239.1249.

(+)-(S,E)-Ethyl 4-hydroxy-4-((S)-hydroxy(phenyl)methyl)pent-2-enoate (11c)After purification by flash column chromatography (50\% EtOAc/hexanes) the reaction yielded $490 \mathrm{mg}(60 \%)$ of diol as a clear, yellow oil. $R_{f}=0.10$ (4:1 hexanes:EtOAc), $[\alpha]_{\mathrm{D}}{ }^{24}+15.7(c$ 1.2, $\mathrm{CH}_{2} \mathrm{Cl}_{2}$ ); IR (neat, $\mathrm{cm}^{-1}$ ) 3436, 2981, 1699, 1656, 1453, 1368, 1304, 1278, 1182, 1094, 1026, 985, 910, 868, 721, 700; ${ }^{1} \mathrm{H}$ NMR $\left(\mathrm{CDCl}_{3}, 600 \mathrm{MHz}\right) \delta 7.33(\mathrm{~m}, 5 \mathrm{H}), 7.01(\mathrm{~d}, J=15.6$ $\mathrm{Hz}, 1 \mathrm{H}), 6.11(\mathrm{~d}, J=15.6 \mathrm{~Hz}, 1 \mathrm{H}), 4.60(\mathrm{~d}, J=3.0 \mathrm{~Hz}, 1 \mathrm{H}), 4.19(\mathrm{q}, J=7.2 \mathrm{~Hz}, 2 \mathrm{H}), 2.70(\mathrm{~s}$, $1 \mathrm{H}), 2.47(\mathrm{~s}, 1 \mathrm{H}), 1.28(\mathrm{t}, J=7.2 \mathrm{~Hz}, 3 \mathrm{H}), 1.17(\mathrm{~s}, 3 \mathrm{H}) ;{ }^{13} \mathrm{C} \mathrm{NMR}\left(\mathrm{CDCl}_{3}, 150 \mathrm{MHz}\right) \delta 166.5$ $151.8,138.9,128.2,128.1$ (2C), 127.5 (2C), 120.5, 79.2, 75.5, 60.4, 22.9, 14.1; HRMS (ESI) calcd for $\left[\mathrm{C}_{14} \mathrm{H}_{18} \mathrm{O}_{4}+\mathrm{Na}\right]^{+}:$273.1097, Found: 273.1091

(+)-( E,4R,5S)-Ethyl 4,5-dihydroxy-4-methylhex-2-enoate (17)-After purification by flash column chromatography (30\% EtOAc/hexanes) the reaction yielded $153 \mathrm{mg}(64 \%)$ of diol as a clear, colorless oil. $R_{f}=0.10$ (4:1 hexanes:EtOAc), $[\alpha]_{\mathrm{D}}{ }^{24}+2.5\left(c 1.00, \mathrm{CH}_{2} \mathrm{Cl}_{2}\right)$; IR (neat, $\mathrm{cm}^{-1}$ ) 3434(br), 2980, 2936, 1699, 1655, 1449, 1368, 1303, 1276, 1181, 1090, 1033, 985, 920, 887, 729; ${ }^{1} \mathrm{H}$ NMR $\left(\mathrm{CDCl}_{3}, 600 \mathrm{MHz}\right) \delta 6.97(\mathrm{~d}, J=15.6 \mathrm{~Hz}, 1 \mathrm{H}), 6.11(\mathrm{~d}, J=15.6$ $\mathrm{Hz}, 1 \mathrm{H}), 4.19$ (q, $J=7.2 \mathrm{~Hz}, 2 \mathrm{H}), 3.71(\mathrm{q}, J=6.6 \mathrm{~Hz}, 1 \mathrm{H}), 2.64$ (brs, 1H), 2.28 (brs, $1 \mathrm{H}), 1.31$ (s, $3 \mathrm{H}), 1.28(\mathrm{t}, J=7.2 \mathrm{~Hz}, 3 \mathrm{H}), 1.17(\mathrm{dd}, J=6.6,1.2 \mathrm{~Hz}, 3 \mathrm{H}) ;{ }^{13} \mathrm{C} \mathrm{NMR}\left(\mathrm{CDCl}_{3}, 150 \mathrm{MHz}\right)$ $\delta$ 166.7, 150.0, 121.2, 75.6, 74.0, 60.7, 24.6, 18.2, 14.4 .

\section{General procedure for carbonate formation}

To diol in $\mathrm{CH}_{2} \mathrm{Cl}_{2}(0.4 \mathrm{M})$ in an ice bath was added pyridine (5 equiv.). Triphosgene (1.1 equiv.) in $\mathrm{CH}_{2} \mathrm{Cl}_{2}(0.4 \mathrm{M}$, total reaction concentration equals $0.2 \mathrm{M})$ was added via syringe and the reaction was allowed to stir for 5 min until determined complete by TLC (UV, PMA stain). The reaction was diluted with diethyl ether and was placed in a separatory funnel. The crude mixture, including salts, was washed vigorously with a saturated aqueous $\mathrm{CuSO}_{4}$ solution until all salts dissolved. The layers were then separated and the organic layer was washed with brine. After separation the organic layer was dried over $\mathrm{Na}_{2} \mathrm{SO}_{4}$ and concentrated. The crude mixture was then purified by flash column chromatography.

(+)-(E)-Ethyl 3-((4S,5S)-5-isobutyl-4-methyl-2-oxo-1,3-dioxolan-4-yl)acrylate (13a)-The crude mixture was purified by flash column chromatography (20\% EtOAc/ 
hexanes) to yield carbonate in $90 \%$ yield as a clear, colorless oil. $R_{f}=0.26$ (4:1 hexanes:EtOAc), $[\alpha]_{\mathrm{D}}{ }^{24}+35.6\left(c 1.0, \mathrm{CH}_{2} \mathrm{Cl}_{2}\right)$; IR (neat, $\left.\mathrm{cm}^{-1}\right) 2961,1799,1719,1663,1468,1385,1367$, $1309,1280,1232,1177,1088,1063,1013,982,870,774 ;{ }^{1} \mathrm{H}$ NMR $\left(\mathrm{CDCl}_{3}, 600 \mathrm{MHz}\right) \delta 6.85$ $(\mathrm{d}, J=15.6 \mathrm{~Hz}, 1 \mathrm{H}), 6.16(\mathrm{~d}, J=15.6 \mathrm{~Hz}, 1 \mathrm{H}), 4.41(\mathrm{dd}, J=10.8,2.4 \mathrm{~Hz}, 1 \mathrm{H}), 4.22(\mathrm{q}, J=$ $7.2 \mathrm{~Hz}, 2 \mathrm{H}), 1.84(\mathrm{~m}, 1 \mathrm{H}), 1.71(\mathrm{~m}, 1 \mathrm{H}), 1.46(\mathrm{~s}, 3 \mathrm{H}), 1.32(\mathrm{~m}, 1 \mathrm{H}), 1.30(\mathrm{t}, J=7.2 \mathrm{~Hz}, 3 \mathrm{H})$, $0.99(\mathrm{~d}, J=6.6 \mathrm{~Hz}, 3 \mathrm{H}), 0.95(\mathrm{~d}, J=7.2 \mathrm{~Hz}, 3 \mathrm{H}) ;{ }^{13} \mathrm{C} \mathrm{NMR}\left(\mathrm{CDCl}_{3}, 150 \mathrm{MHz}\right) \delta 165.3,153.2$, 144.0, 122.3, 84.15, 82.0, 61.0, 37.4, 25.1, 23.1, 21.5, 19.2, 14.1; HRMS (ESI) calcd for $\left[\mathrm{C}_{13} \mathrm{H}_{20} \mathrm{O}_{5}+\mathrm{Na}\right]^{+}:$279.1202, Found: 279.1204 .

(+)-(E)-Ethyl 3-((4S,5S)-5-isopropyl-4-methyl-2-oxo-1,3-dioxolan-4-yl)acrylate (13b)-The crude mixture was purified by flash column chromatography (20\% EtOAc/ hexanes) to yield carbonate in $84 \%$ yield as a clear, colorless oil. $R_{f}=0.22$ (4:1 hexanes:EtOAc), $[\alpha]_{\mathrm{D}}{ }^{24}+18.3\left(c 1.0, \mathrm{CH}_{2} \mathrm{Cl}_{2}\right)$; IR (neat, $\left.\mathrm{cm}^{-1}\right) 2973,1801,1720,1663,1472,1368,1281,1245$, $1175,1109,1062,1027,982,839,774 ;{ }^{1} \mathrm{H}$ NMR $\left(\mathrm{CDCl}_{3}, 600 \mathrm{MHz}\right) \delta 6.84(\mathrm{~d}, J=15.6 \mathrm{~Hz}$, $1 \mathrm{H}), 6.20(\mathrm{~d}, J=15.6 \mathrm{~Hz}, 1 \mathrm{H}), 4.23(\mathrm{q}, J=7.2 \mathrm{~Hz}, 2 \mathrm{H}), 3.98(\mathrm{~d}, J=9.6 \mathrm{~Hz}, 1 \mathrm{H}), 2.04(\mathrm{~m}, 1 \mathrm{H})$, $1.54(\mathrm{~s}, 3 \mathrm{H}), 1.30(\mathrm{t}, J=7.2 \mathrm{~Hz}, 3 \mathrm{H}), 1.11(\mathrm{~d}, J=6.6 \mathrm{~Hz}, 3 \mathrm{H}), 0.95(\mathrm{~d}, J=6.6 \mathrm{~Hz}, 3 \mathrm{H}) ;{ }^{13} \mathrm{C}$ $\mathrm{NMR}\left(\mathrm{CDCl}_{3}, 150 \mathrm{MHz}\right) \delta 165.6,153.1,144.5,123.0,89.1,84.5,61.2,28.2,19.8,19.1,18.7$, 14.3; HRMS (ESI) calcd for $\left[\mathrm{C}_{12} \mathrm{H}_{18} \mathrm{O}_{5}+\mathrm{Na}\right]^{+}: 265.1046$, Found: 265.1050.

\section{(-)-(E)-Ethyl 3-((4S,5S)-4-methyl-2-oxo-5-phenyl-1,3-dioxolan-4-yl)acrylate}

(13c)-The crude mixture was purified by flash column chromatography (20\% EtOAc/ hexanes) to yield carbonate in $91 \%$ yield as a clear, colorless oil. $R_{f}=0.21$ ( $4: 1$ hexanes:EtOAc), $[\alpha]_{\mathrm{D}}{ }^{24}-24.9\left(c 1.3, \mathrm{CH}_{2} \mathrm{Cl}_{2}\right)$; IR (neat, $\left.\mathrm{cm}^{-1}\right) 2984,1802,1718,1663,1456,1367,1308,1288$, 1245, 1180, 1088, 1069, 1044, 1028, 979, 771, 700; ${ }^{1} \mathrm{H}$ NMR $\left(\mathrm{CDCl}_{3}, 600 \mathrm{MHz}\right) \delta 7.43(\mathrm{~m}$, $3 \mathrm{H}), 7.28(\mathrm{~m}, 2 \mathrm{H}), 7.01(\mathrm{~d}, J=15.6 \mathrm{~Hz}, 1 \mathrm{H}), 6.20(\mathrm{~d}, J=15.6 \mathrm{~Hz}, 1 \mathrm{H}), 5.47(\mathrm{~s}, 1 \mathrm{H}), 4.26(\mathrm{qd}$, $J=7.2,1.2 \mathrm{~Hz}, 2 \mathrm{H}), 1.32(\mathrm{t}, J=7.2 \mathrm{~Hz}, 3 \mathrm{H}), 1.12(\mathrm{~s}, 3 \mathrm{H}) ;{ }^{13} \mathrm{C} \mathrm{NMR}\left(\mathrm{CDCl}_{3}, 150 \mathrm{MHz}\right) \delta$ 165.3, 153.0, 144.5, 132.2, 129.5, 128.9 (2C), 125.5 (2C), 122.6, 85.2, 84.4, 61.1, 20.9, 14.1; HRMS (ESI) calcd for $\left[\mathrm{C}_{15} \mathrm{H}_{16} \mathrm{O}_{5}+\mathrm{Na}\right]^{+}: 299.0889$, Found: 299.0897.

(+)-(E)-Ethyl 3-((4S,5S)-4,5-dimethyl-2-oxo-1,3-dioxolan-4-yl)acrylate (13d)-The crude mixture was purified by flash column chromatography (20\% EtOAc/hexanes) to yield carbonate in $90 \%$ yield as a clear, colorless oil. $R_{f}=0.25$ (4:1 hexanes:EtOAc), $[\alpha]_{\mathrm{D}}{ }^{24}+12.7$ (c 1.00, $\mathrm{CH}_{2} \mathrm{Cl}_{2}$ ); IR (neat, cm ${ }^{-1}$ ) 2987, 2941, 2301, 1821, 1726, 1664, 1446, 1390, 1348, 1312, $1235,1183,1086,1034,868,774,630 ;{ }^{1} \mathrm{H} \mathrm{NMR}\left(\mathrm{CDCl}_{3}, 600 \mathrm{MHz}\right) \delta 6.86(\mathrm{~d}, J=16.2 \mathrm{~Hz}$, $1 \mathrm{H}), 6.15(\mathrm{~d}, J=16.2 \mathrm{~Hz}, 1 \mathrm{H}), 4.54(\mathrm{q}, J=6.6 \mathrm{~Hz}, 1 \mathrm{H}), 4.22(\mathrm{q}, J=6.6 \mathrm{~Hz}, 2 \mathrm{H}), 1.48(\mathrm{~s}, 3 \mathrm{H})$, $1.41(\mathrm{~d}, J=6.6 \mathrm{~Hz}, 3 \mathrm{H}), 1.30(\mathrm{t}, J=6.6 \mathrm{~Hz}, 3 \mathrm{H}) ;{ }^{13} \mathrm{C} \mathrm{NMR}\left(\mathrm{CDCl}_{3}, 150 \mathrm{MHz}\right) \delta 165.3,153.1$, 144.0, 122.3, 84.1, 79.6, 61.0, 19.1, 14.3, 14.1; HRMS (ESI) calcd for $\left[\mathrm{C}_{10} \mathrm{H}_{14} \mathrm{O}_{5}+\mathrm{Na}\right]^{+}$: 237.0733, Found: 237.0726.

(-)-(E)-Ethyl 3-((4S,5S)-5-(2-(tert-butyldimethylsilyloxy)ethyl)-4-methyl-2oxo-1,3-dioxolan-4-yl)acrylate (13f) - The crude mixture was purified by flash column chromatography (20\% EtOAc/hexanes) to yield carbonate in $71 \%$ yield as a clear, colorless oil. $R_{f}=0.50$ (4:1 hexanes:EtOAc), $[\alpha]_{\mathrm{D}}{ }^{24}-52.2\left(c 1.00, \mathrm{CH}_{2} \mathrm{Cl}_{2}\right)$; IR (neat) 2956, 2858, 1813, 1723, 1665, 1469, 1388, 1309, 1258, 1179, 1088, 1033, 982, 835, 777, 721; ${ }^{1} \mathrm{H}$ NMR $\left(\mathrm{CDCl}_{3}, 600 \mathrm{MHz}\right) \delta 6.89(\mathrm{~d}, J=15.6 \mathrm{~Hz}, 1 \mathrm{H}), 6.15(\mathrm{~d}, J=15.6 \mathrm{~Hz}, 1 \mathrm{H}), 4.59(\mathrm{dd}, J=9.6$, $3.6 \mathrm{~Hz}, 1 \mathrm{H}), 4.22(\mathrm{q}, J=7.2 \mathrm{~Hz}, 2 \mathrm{H}), 3.77(\mathrm{~m}, 2 \mathrm{H}), 1.87(\mathrm{~m}, 2 \mathrm{H}), 1.49(\mathrm{~s}, 3 \mathrm{H}), 1.30(\mathrm{t}, J=7.2$ $\mathrm{Hz}, 3 \mathrm{H}), 0.88$ (s, 9H), 0.06 (s, 3H), $0.06(\mathrm{~s}, 3 \mathrm{H}) ;{ }^{13} \mathrm{C} \mathrm{NMR}\left(\mathrm{CDCl}_{3}, 150 \mathrm{MHz}\right) \delta 165.3,153.1$, 144.1, 122.2, 83.9, 80.3, 61.0, 58.5, 32.1, 25.8 (3C), 19.6, 18.2, 14.1, -5.5 (2C); HRMS (ESI) calcd for $\left[\mathrm{C}_{17} \mathrm{H}_{30} \mathrm{O}_{6} \mathrm{Si}+\mathrm{Na}\right]^{+}: 381.1703$, Found: 381.1718 .

(-)-(E)-Ethyl 3-((4S,5S)-5-(2-(benzyloxy)ethyl)-4-methyl-2-oxo-1,3-dioxolan-4-yl) acrylate $\mathbf{( 1 3 g )}$ - The crude mixture was purified by flash column chromatography (20\% 
EtOAc/hexanes) to yield carbonate in $88 \%$ yield as a clear, colorless oil. $R_{f}=0.23$ ( $4: 1$ hexanes:EtOAc), $[\alpha]_{\mathrm{D}}{ }^{24}-23.8$ ( 2 2.3, $\left.\mathrm{CH}_{2} \mathrm{Cl}_{2}\right)$; IR (neat, $\left.\mathrm{cm}^{-1}\right)$ 2985, 2863, 1810, 1720, 1662 , $1556,1495,1454,1382,1188,1090,985,773,741 ; ;{ }^{1} \mathrm{H} \mathrm{NMR}\left(\mathrm{CDCl}_{3}, 600 \mathrm{MHz}\right) \delta 7.35(\mathrm{~m}$, $3 \mathrm{H}), 7.31(\mathrm{~m}, 2 \mathrm{H}), 6.88(\mathrm{~d}, J=15.6 \mathrm{~Hz}, 1 \mathrm{H}), 6.15(\mathrm{~d}, J=15.6 \mathrm{~Hz}, 1 \mathrm{H}), 4.62(\mathrm{t}, J=7.2 \mathrm{~Hz}$, $1 \mathrm{H}), 4.51(\mathrm{dd}, J=27.0,6.0 \mathrm{~Hz}, 2 \mathrm{H}), 4.22(\mathrm{q}, J=7.2 \mathrm{~Hz}, 2 \mathrm{H}), 3.63(\mathrm{~m}, 2 \mathrm{H}), 1.95(\mathrm{dd}, J=12.6$, $6.0 \mathrm{~Hz}, 2 \mathrm{H}), 1.48(\mathrm{~s}, 3 \mathrm{H}), 1.30(\mathrm{t}, J=7.2 \mathrm{~Hz}, 3 \mathrm{H}) ;{ }^{13} \mathrm{C} \mathrm{NMR}\left(\mathrm{CDCl}_{3}, 150 \mathrm{MHz}\right) \delta 165.3,153.0$, 144.1, 137.5, 128.4 (2C), 127.8, 127.7 (2C), 122.2, 84.0, 80.5, 73.4, 65.4, 61.0, 29.6, 19.5, 14.1; HRMS (ESI) calcd for $\left[\mathrm{C}_{18} \mathrm{H}_{22} \mathrm{O}_{6}+\mathrm{Na}\right]^{+}: 357.1308$, Found: 357.1290 .

(-)-(E)-Ethyl 3-((4R,5S)-4,5-dimethyl-2-oxo-1,3-dioxolan-4-yl)acrylate (18)-The crude mixture was purified by flash column chromatography (20\% EtOAc/hexanes) to yield carbonate in $89 \%$ yield as a clear, colorless oil. $R_{f}=0.20$ (4:1 hexanes:EtOAc), $[\alpha]_{\mathrm{D}}{ }^{24}-30.2$ (c 0.65, $\mathrm{CH}_{2} \mathrm{Cl}_{2}$ ); IR (neat, $\mathrm{cm}^{-1}$ ) 2985, 2940, 1793, 1717, 1662, 1594, 1448, 1387, 1367, 1310, $1287,1225,1180,1097,1074,1000,905,870,773,732,685 ;{ }^{1} \mathrm{H} \mathrm{NMR}\left(\mathrm{CDCl}_{3}, 600 \mathrm{MHz}\right) \delta$ $6.76(\mathrm{~d}, J=15.6 \mathrm{~Hz}, 1 \mathrm{H}), 6.19(\mathrm{~d}, J=15.6 \mathrm{~Hz}, 1 \mathrm{H}), 4.55(\mathrm{dd}, J=6.6,6.6 \mathrm{~Hz}, 1 \mathrm{H}), 4.22(\mathrm{q}$, $J=7.2 \mathrm{~Hz}, 2 \mathrm{H}), 1.60(\mathrm{~s}, 3 \mathrm{H}), 1.32(\mathrm{~d}, J=6.6 \mathrm{~Hz}, 3 \mathrm{H}), 1.30(\mathrm{t}, J=7.2 \mathrm{~Hz}, 3 \mathrm{H}) ;{ }^{13} \mathrm{C}$ NMR $\left(\mathrm{CDCl}_{3}, 150 \mathrm{MHz}\right) \delta 165.4,153.3,142.1,123.2,84.4,81.9,61.2,24.4,16.1,14.3$; HRMS (ESI) calcd for $\left[\mathrm{C}_{10} \mathrm{H}_{14} \mathrm{O}_{5}+\mathrm{Na}\right]^{+}:$237.0733, Found: 237.0736.

\section{General procedure for the $\mathrm{Pd}$-catalyzed carbonate reduction}

To a flask containing carbonate in THF $(0.2 \mathrm{M})$ was added $\mathrm{Pd}_{2}(\mathrm{dba})_{3} \cdot \mathrm{CHCl}_{3}(1 \mathrm{~mol} \%)$, $\mathrm{PPh}_{3}(1 \mathrm{~mol} \%), \mathrm{Et}_{3} \mathrm{~N}$ (5 equiv.) and finally formic acid (5 equiv.). The reaction was then refluxed for 20-40 min at which time it was determined complete by TLC (UV, anisaldehyde). The reaction was then allowed to cool to RT then diluted with ether and filtered through a plug of silica gel to remove $\operatorname{Pd}(0)$ before concentration. The crude mixture was then concentrated and subjected to flash column chromatography.

\section{(-)-(E,4S,5S)-Ethyl 7-(tert-butyldimethylsilyloxy)-5-hydroxy-4-methylhept-2- enoate (8a)-The crude mixture was concentrated and subjected to flash column chromatography (20\% EtOAc/hexanes) to yield $\delta$-hydroxy enoate in $95 \%$ yield as a clear, colorless oil. $R_{f}=0.45$ (4:1 hexanes:EtOAc), $[\alpha]_{\mathrm{D}}{ }^{24}-16.5\left(c 0.63, \mathrm{CH}_{2} \mathrm{Cl}_{2}\right.$; IR (neat, cm) 3499 (br), 2955, 2859, 1720, 1651, 1463, 1368, 1256, 1181, 1144, 1093, 1038, 986, 835, 777, 726; ${ }^{1} \mathrm{H}$ NMR $\left(\mathrm{CDCl}_{3}, 600 \mathrm{MHz}\right) \delta 6.93(\mathrm{dd}, J=16.2,7.8 \mathrm{~Hz}, 1 \mathrm{H}), 5.84(\mathrm{dd}, J=16.2,1.2 \mathrm{~Hz}$, $1 \mathrm{H}), 4.17(\mathrm{q}, J=7.2 \mathrm{~Hz}, 2 \mathrm{H}), 3.89(\mathrm{~m}, 1 \mathrm{H}), 3.78(\mathrm{~m}, 2 \mathrm{H}), 2.41(\mathrm{~m}, 1 \mathrm{H}), 1.61(\mathrm{~m}, 2 \mathrm{H}), 1.27(\mathrm{t}$, $J=7.2 \mathrm{~Hz}, 3 \mathrm{H}), 1.10(\mathrm{~d}, J=7.2 \mathrm{~Hz}, 3 \mathrm{H}), 0.88(\mathrm{~s}, 9 \mathrm{H}), 0.07(\mathrm{~d}, J=1.2 \mathrm{~Hz}, 6 \mathrm{H}) ;{ }^{13} \mathrm{C}$ NMR $\left(\mathrm{CDCl}_{3}, 150 \mathrm{MHz}\right) \delta 166.6,150.9,121.4,75.1,63.0,60.2,42.7,35.4,25.8$ (3C), 18.0, 14.7, 14.2, -5.6 (2C); HRMS (ESI) calcd for $\left[\mathrm{C}_{16} \mathrm{H}_{32} \mathrm{O}_{4} \mathrm{Si}+\mathrm{Na}\right]^{+}: 339.1962$, Found: 339.1954.}

(-)-(E,4S,5S)-Ethyl 7-(benzyloxy)-5-hydroxy-4-methylhept-2-enoate (8b)-The crude mixture was concentrated and subjected to flash column chromatography $(20 \% \mathrm{EtOAc} /$ hexanes) to yield $\delta$-hydroxy enoate in $96 \%$ yield as a clear, colorless oil. $R_{f}=0.23$ ( $4: 1$ hexanes:EtOAc), $[\alpha]_{\mathrm{D}}{ }^{24}-18.9\left(c 1.20, \mathrm{CH}_{2} \mathrm{Cl}_{2}\right)$; IR (neat, $\left.\mathrm{cm}^{-1}\right)$ 3486(br), 2932, 2854, 1725, 1646, 1467, 1273, 1193, 1107, 1090, 741, 705; ${ }^{1} \mathrm{H}$ NMR $\left(\mathrm{CDCl}_{3}, 600 \mathrm{MHz}\right) \delta 7.31(\mathrm{~m}, 5 \mathrm{H})$, $6.95(\mathrm{dd}, J=15.6,7.8 \mathrm{~Hz}, 1 \mathrm{H}), 5.84(\mathrm{dd}, J=15.6,1.8 \mathrm{~Hz}, 1 \mathrm{H}), 4.51(\mathrm{~s}, 2 \mathrm{H}), 4.18(\mathrm{q}, J=7.2$ $\mathrm{Hz}, 2 \mathrm{H}), 3.74$ (m, 2H), 3.64 (ddd, $J=12.6,6.6,2.4 \mathrm{~Hz}, 1 \mathrm{H}), 3.01$ (d, $J=3.0 \mathrm{~Hz}, 1 \mathrm{H}), 2.43$ (sextet, $J=6.6 \mathrm{~Hz}, 1 \mathrm{H}), 1.73(\mathrm{q}, J=6.0 \mathrm{~Hz}, 2 \mathrm{H}), 1.29(\mathrm{t}, J=7.2 \mathrm{~Hz}, 3 \mathrm{H}), 1.11(\mathrm{~d}, J=7.2 \mathrm{~Hz}$, $3 \mathrm{H}) ;{ }^{13} \mathrm{C} \mathrm{NMR}\left(\mathrm{CDCl}_{3}, 150 \mathrm{MHz}\right) \delta 166.5,150.7,137.7,128.4$ (2C), 127.8, 127.7 (2C), 121.5, 74.4, 73.4, 69.4, 60.2, 42.6, 33.6, 14.6, 14.2; HRMS (ESI) calcd for $\left[\mathrm{C}_{17} \mathrm{H}_{24} \mathrm{O}_{4} \mathrm{Si}+\mathrm{Na}\right]^{+}$: 315.1566, Found: 315.1568 .

(-)-(E,4S,5S)-ethyl 5-hydroxy-4,7-dimethyloct-2-enoate (14a)-The crude mixture was concentrated and subjected to flash column chromatography (20\% EtOAc/hexanes) to 
yield $\delta$-hydroxy enoate in $96 \%$ yield as a clear, colorless oil. $R_{f}=0.27$ (4:1 hexanes:EtOAc), $[\alpha]_{\mathrm{D}}{ }^{24}-43.7$ (c 1.0, $\mathrm{CH}_{2} \mathrm{Cl}_{2}$ ); IR (neat, $\mathrm{cm}^{-1}$ ) 3436(br), 2957, 2871, 1702, 1651, 1467, 1368, $1272,1182,1150,1095,1034,989,865,729 ;{ }^{1} \mathrm{H}$ NMR $\left(\mathrm{CDCl}_{3}, 600 \mathrm{MHz}\right) \delta 6.95(\mathrm{dd}, J=$ 15.6, $7.8 \mathrm{~Hz}, 1 \mathrm{H}), 5.86(\mathrm{dd}, J=15.6,1.8 \mathrm{~Hz}, 1 \mathrm{H}), 4.21(\mathrm{q}, J=7.2 \mathrm{~Hz}, 2 \mathrm{H}), 3.67$ (ddd, $J=9.0$, 8.4, $5.4 \mathrm{~Hz}, 1 \mathrm{H}$ ), 2.40 (sextet, $J=7.2 \mathrm{~Hz}, 1 \mathrm{H}), 1.77$ (m, 2H), 1.57 (brs, $1 \mathrm{H}), 1.35$ (m, $1 \mathrm{H}), 1.29$ $(\mathrm{t}, J=7.2 \mathrm{~Hz}, 3 \mathrm{H}), 1.08(\mathrm{~d}, J=7.2 \mathrm{~Hz}, 3 \mathrm{H}), 0.94(\mathrm{~d}, J=6.6 \mathrm{~Hz}, 3 \mathrm{H}), 0.90(\mathrm{~d}, J=6.6 \mathrm{~Hz}$, $3 \mathrm{H}) ;{ }^{13} \mathrm{C} \mathrm{NMR}\left(\mathrm{CDCl}_{3}, 150 \mathrm{MHz}\right) \delta 166.5,150.9,121.6,72.4,60.2,43.4,42.8,24.6,23.6$, 21.6, 14.2, 13.8; HRMS (ESI) calcd for $\left[\mathrm{C}_{12} \mathrm{H}_{22} \mathrm{O}_{3}+\mathrm{Na}\right]^{+}:$: 237.1461, Found: 237.1460.

(-)-(E,4S,5S)-Ethyl 5-hydroxy-4-methylhex-2-enoate (14d)-The crude mixture was concentrated and subjected to flash column chromatography (20\% EtOAc/hexanes) to yield $\delta$-hydroxy enoate in $98 \%$ yield as a clear, colorless oil. $R_{f}=0.40$ ( $4: 1$ hexanes:EtOAc), $[\alpha]_{\mathrm{D}}{ }^{24}-29.8\left(c 0.77, \mathrm{CH}_{2} \mathrm{Cl}_{2}\right.$ ); IR (neat, $\left.\mathrm{cm}^{-1}\right)$ 3410(br), 2971, 2850, 1716, 1650, 1273, 1183, $1155,1093,1034 ;{ }^{1} \mathrm{H}$ NMR $\left(\mathrm{CDCl}_{3}, 600 \mathrm{MHz}\right) \delta 6.93(\mathrm{dd}, J=15.6,7.8 \mathrm{~Hz}, 1 \mathrm{H}), 5.86(\mathrm{dd}, J$ $=15.6,1.2 \mathrm{~Hz}, 1 \mathrm{H}), 4.18(\mathrm{q}, J=7.2 \mathrm{~Hz}, 2 \mathrm{H}), 3.77(\mathrm{~m}, 1 \mathrm{H}), 2.39(\mathrm{~m}, 1 \mathrm{H}), 1.58$ (brs, $1 \mathrm{H}), 1.29$ $(\mathrm{t}, J=7.2 \mathrm{~Hz}, 3 \mathrm{H}), 1.16(\mathrm{~d}, J=6.6 \mathrm{~Hz}, 3 \mathrm{H}), 1.08(\mathrm{~d}, J=6.6 \mathrm{~Hz}, 3 \mathrm{H}) ;{ }^{13} \mathrm{C} \mathrm{NMR}\left(\mathrm{CDCl}_{3}, 150\right.$ MHz) $\delta 166.5,150.4,121.8,70.5,60.2,43.7,20.4,14.4,14.2$; HRMS (ESI) calcd for $\left[\mathrm{C}_{9} \mathrm{H}_{16} \mathrm{O}_{3}+\mathrm{Na}\right]^{+}:$195.0991, Found: 195.1000 .

\section{General procedure for the Evans' hemiacetal addition}

Enoate was dissolved in THF $(0.2 \mathrm{M})$ and cooled to $0{ }^{\circ} \mathrm{C}$. To the solution was added benzaldehyde ( 1.1 equiv.) and potassium tert-butoxide ( 0.15 equiv.). The addition of base and aldehyde was repeated 3 times at 20 minute intervals. The reaction was allowed to stir at $0^{\circ}$ $\mathrm{C}$, and was quenched after 1 hour by adding $\mathrm{pH} 7$ buffered phosphate solution. The layers were separated and the aqueous layer was extracted with $\mathrm{Et}_{2} \mathrm{O}$. The organic layers were combined and washed with brine, then dried over $\mathrm{Na}_{2} \mathrm{SO}_{4}$, and the solvent removed under reduced pressure. The product was purified by silica gel chromatography.

\section{(-)-Ethyl 2-((2S,4R,5R,6S)-6-(2-(tertbutyldimethylsilyloxy)ethyl)-5-methyl-2-} phenyl-1,3-dioxan-4-yl)acetate (9a)-The product was purified by silica gel chromatography eluting with $5 \%$ EtOAc/hexanes to yield benzylidine acetal in 58\% yield as a clear, colorless oil. $R_{f}=0.62$ (4:1 hexanes:EtOAc), $[\alpha]_{\mathrm{D}}{ }^{24}-27.5\left(c 0.50, \mathrm{CH}_{2} \mathrm{Cl}_{2}\right)$; IR (neat, $\mathrm{cm}^{-1}$ ) 2955, 2930, 2858, 1738, 1461, 1390, 1349, 1314, 1254, 1182, 1098, 1064, 1027, 941, 835, 776, 697; ${ }^{1} \mathrm{H}$ NMR $\left(\mathrm{CDCl}_{3}, 600 \mathrm{MHz}\right) \delta 7.49(\mathrm{~m}, 2 \mathrm{H}), 7.34(\mathrm{~m}, 3 \mathrm{H}), 5.58(\mathrm{~s}, 1 \mathrm{H}), 4.43$ (ddd, $J=7.8,6.0,2.4 \mathrm{~Hz}, 1 \mathrm{H}), 4.16(\mathrm{dq}, J=7.2,1.2 \mathrm{~Hz}, 2 \mathrm{H}), 4.13(\mathrm{ddd}, J=8.4,3.0,3.0 \mathrm{~Hz}$, $1 \mathrm{H}), 3.78(\mathrm{~m}, 1 \mathrm{H}), 3.73(\mathrm{~m}, 1 \mathrm{H}), 2.71(\mathrm{dd}, J=15.6,8.4 \mathrm{~Hz}, 1 \mathrm{H}), 2.48(\mathrm{dd}, J=15.6,6.0 \mathrm{~Hz}$, $1 \mathrm{H}), 1.87(\mathrm{~m}, 1 \mathrm{H}), 1.66(\mathrm{~m}, 1 \mathrm{H}), 1.58(\mathrm{qdd}, J=7.2,2.4,2.4 \mathrm{~Hz}, 1 \mathrm{H}), 1.26(\mathrm{t}, J=7.2 \mathrm{~Hz}, 3 \mathrm{H})$, $1.01(\mathrm{~d}, J=7.2, \mathrm{~Hz}, 3 \mathrm{H}), 0.91(\mathrm{~d}, J=1.2 \mathrm{~Hz}, 9 \mathrm{H}), 0.06(\mathrm{~d}, J=6.4 \mathrm{~Hz}, 6 \mathrm{H}) ;{ }^{13} \mathrm{C}$ NMR $\left(\mathrm{CDCl}_{3}, 150 \mathrm{MHz}\right) \delta 171.0,138.6,128.6,128.1$ (2C), 126.1 (2C), 101.5, 77.3, 77.1, 60.5, 59.2, 38.1, 35.8, 34.6, 25.9 (3C), 18.3, 14.1, 6.2, -5.3(2C); HRMS (ESI) calcd for $\left[\mathrm{C}_{23} \mathrm{H}_{38} \mathrm{O}_{5}+\right.$ $\mathrm{Na}]^{+}:$445.2380, Found: 445.2398 .

\section{(-)-Ethyl 2-((2S,4R,5R,6S)-6-(2-(benzyloxy)ethyl)-5-methyl-2-phenyl-1,3-}

dioxan-4-yl)acetate (9b)-The product was purified by silica gel chromatography eluting with $5 \%$ EtOAc/hexanes to yield benzylidine acetal in $69 \%$ yield as a clear, colorless oil. $R_{f}$ $=0.39$ (4:1 hexanes:EtOAc), $[\alpha]_{\mathrm{D}}{ }^{24}-20.5\left(c 1.0, \mathrm{CH}_{2} \mathrm{Cl}_{2}\right) ; \mathrm{IR}\left(\right.$ neat, $\left.\mathrm{cm}^{-1}\right) 2978,2869,1735$, 1496, 1454, 1369, 1350, 1264, 1182, 1151, 1100, 1066, 1027, 754, 697; ${ }^{1} \mathrm{H} \mathrm{NMR}\left(\mathrm{CDCl}_{3}, 600\right.$ $\mathrm{MHz}) \delta 7.33(\mathrm{~m}, 10 \mathrm{H}), 5.5(\mathrm{~s}, 1 \mathrm{H}), 4.54(\mathrm{~d}, J=12.0 \mathrm{~Hz}, 1 \mathrm{H}), 4.50(\mathrm{~d}, J=12.0, \mathrm{~Hz}, 1 \mathrm{H}), 4.42$ (ddd, $J=8.4,5.4,2.4 \mathrm{~Hz}, 1 \mathrm{H}), 4.18$ (qd, $J=7.2,1.8 \mathrm{~Hz}, 1 \mathrm{H}), 4.17$ (ddd, $J=8.4,3.6,2.4 \mathrm{~Hz}$, $1 \mathrm{H}), 4.15$ (qd, $J=7.2,1.8 \mathrm{~Hz}, 1 \mathrm{H}$ ), 3.65 (ddd, $J=13.2,9.0,4.8 \mathrm{~Hz}, 1 \mathrm{H}$ ), 3.59 (ddd, $J=12.0$, 9.0, $6.0 \mathrm{~Hz}, 1 \mathrm{H}), 2.71(\mathrm{dd}, J=15.6,7.8 \mathrm{~Hz}, 1 \mathrm{H}), 2.47(\mathrm{dd}, J=15.6,5.4 \mathrm{~Hz}, 1 \mathrm{H}), 1.97(\mathrm{~m}, 1 \mathrm{H})$, $1.76(\mathrm{~m}, 1 \mathrm{H}), 1.58(\mathrm{qdd}, J=6.6,2.4,2.4 \mathrm{~Hz}, 1 \mathrm{H}), 1.27(\mathrm{t}, J=7.2 \mathrm{~Hz}, 3 \mathrm{H}), 1.01(\mathrm{~d}, J=6.6 \mathrm{~Hz}$, 
$3 \mathrm{H}) ;{ }^{13} \mathrm{C} \mathrm{NMR}\left(\mathrm{CDCl}_{3}, 150 \mathrm{MHz}\right) \delta 170.9,138.6,138.4,128.6,128.3(2 \mathrm{C}), 128.1(2 \mathrm{C}), 127.6$ (2C), 127.5, 126.1 (2C), 101.4, 77.5, 77.2, 73.0. 66.4, 60.5, 38.1, 34.5, 33.1, 14.1, 6.1; HRMS (ESI) calcd for $\left[\mathrm{C}_{24} \mathrm{H}_{30} \mathrm{O}_{5}+\mathrm{Na}\right]^{+}: 421.1985$, Found: 421.2014.

(-)-Ethyl 2-((2S,4R,5R,6S)-6-isobutyl-5-methyl-2-phenyl-1,3-dioxan-4-yl)acetate (15a)-The product was purified by silica gel chromatography eluting with 5-10\% EtOAc/ hexanes to yield benzylidine acetal in 75\% yield as a clear, colorless oil. $R_{f}=0.39$ ( $4: 1$ hexanes:EtOAc), $[\alpha]_{\mathrm{D}}{ }^{24}-7.3\left(c 0.50, \mathrm{CH}_{2} \mathrm{Cl}_{2}\right)$; IR (neat, $\left.\mathrm{cm}^{-1}\right)$ 2955, 2870, 1734, 1456, 1391, $1368,1349,1259,1180,1102,1056,1021,993,922,853,755,696 ;{ }^{1} \mathrm{H} \mathrm{NMR}\left(\mathrm{CDCl}_{3}, 600\right.$ $\mathrm{MHz}) \delta 7.48(\mathrm{~m}, 2 \mathrm{H}), 7.33(\mathrm{~m}, 3 \mathrm{H}), 5.58(\mathrm{~s}, 1 \mathrm{H}), 4.42(\mathrm{ddd}, J=7.8,5.4,2.4 \mathrm{~Hz}, 1 \mathrm{H}), 4.16(\mathrm{q}$, $J=7.2 \mathrm{~Hz}, 2 \mathrm{H}), 3.99$ (ddd, $J=8.4,4.8,2.4 \mathrm{~Hz}, 1 \mathrm{H}), 2.71(\mathrm{dd}, J=15.6,7.8 \mathrm{~Hz}, 1 \mathrm{H}), 2.48(\mathrm{dd}$, $J=15.6,6.0 \mathrm{~Hz}, 1 \mathrm{H}), 1.78(\mathrm{~m}, 1 \mathrm{H}), 1.64(\mathrm{~m}, 1 \mathrm{H}), 1.54(\mathrm{qdd}, J=7.2,2.4,2.4 \mathrm{~Hz}, 1 \mathrm{H}), 1.27(\mathrm{t}$, $J=7.2 \mathrm{~Hz}, 3 \mathrm{H}), 1.26(\mathrm{~m}, 1 \mathrm{H}), 0.99(\mathrm{~d}, J=7.2 \mathrm{~Hz}, 3 \mathrm{H}), 0.95(\mathrm{~d}, J=6.6 \mathrm{~Hz}, 6 \mathrm{H}) ;{ }^{13} \mathrm{C}$ NMR $\left(\mathrm{CDCl}_{3}, 150 \mathrm{MHz}\right) \delta 171.1,138.7,128.5,128.1$ (2C), 126.0 (2C), 101.4, 79.0, 77.4, 60.5, 41.5, 38.1, 34.6, 24.3, 23.0, 22.6, 14.2, 6.0; HRMS (ESI) calcd for $\left[\mathrm{C}_{19} \mathrm{H}_{28} \mathrm{O}_{4}+\mathrm{Na}\right]^{+}$: 343.1879, Found: 343.1880 .

\section{(+)-Ethyl 2-((2R,4R,5S,6R)-5-methyl-2,6-diphenyl-1,3-dioxan-4-yl)acetate (15c)}

-The product was purified by silica gel chromatography eluting with $10 \%$ EtOAc/hexanes to yield benzylidine acetal in $33 \%$ yield as a clear, colorless oil. $R_{f}=0.26$ ( $4: 1$ hexanes:EtOAc), $[\alpha]_{\mathrm{D}}{ }^{24}+11.1\left(c 1.0, \mathrm{CH}_{2} \mathrm{Cl}_{2}\right)$; IR (neat, $\left.\mathrm{cm}^{-1}\right) 2982,2165,1734,1497,1452,1348,1260,1183$, $1135,1100,1052,1027,993,755,698 ;{ }^{1} \mathrm{H} \mathrm{NMR}\left(\mathrm{CDCl}_{3}, 600 \mathrm{MHz}\right) \delta 7.59(\mathrm{~d}, J=7.2 \mathrm{~Hz}$, $1 \mathrm{H}), 7.37(\mathrm{~m}, 9 \mathrm{H}), 5.79(\mathrm{~s}, 1 \mathrm{H}), 5.15(\mathrm{~d}, J=2.4 \mathrm{~Hz}, 1 \mathrm{H}), 4.65$ (ddd, $J=7.8,6.0,2.4 \mathrm{~Hz}, 1 \mathrm{H})$, 4.19 (q, $J=7.2 \mathrm{~Hz}, 2 \mathrm{H}), 2.76(\mathrm{dd}, J=15.6,8.4 \mathrm{~Hz}, 1 \mathrm{H}), 2.53(\mathrm{dd}, J=15.6,6.0 \mathrm{~Hz}, 1 \mathrm{H}), 1.97$ (qdd, $J=6.6,2.4,2.4 \mathrm{~Hz}, 1 \mathrm{H}), 1.28(\mathrm{t}, J=7.2 \mathrm{~Hz}, 3 \mathrm{H}), 0.79(\mathrm{~d}, J=6.6 \mathrm{~Hz}, 3 \mathrm{H}) ;{ }^{13} \mathrm{C}$ NMR $\left(\mathrm{CDCl}_{3}, 150 \mathrm{MHz}\right) \delta 170.9,140.1,138.5,128.8,128.1(2 \mathrm{C}), 128.1(2 \mathrm{C}), 127.0,126.2(2 \mathrm{C})$, 125.3 (2C), 101.5, 81.7, 77.1, 60.6, 38.2, 36.6, 14.2, 6.1; HRMS (ESI) calcd for $\left[\mathrm{C}_{21} \mathrm{H}_{24} \mathrm{O}_{4}+\right.$ $\mathrm{Na}]^{+}: 363.1566$, Found: 363.1562 .

\section{(+)-Ethyl 2-((2S,4R,5R,6S)-5,6-dimethyl-2-phenyl-1,3-dioxan-4-yl)acetate (15d)}

-The product was purified by silica gel chromatography eluting with $10 \%$ EtOAc/hexanes to yield benzylidine acetal in $63 \%$ yield as a clear, colorless oil. $R_{f}=0.42$ (9:1 hexanes:EtOAc), $[\alpha]_{\mathrm{D}}{ }^{24}+21.6\left(c 1.00, \mathrm{CH}_{2} \mathrm{Cl}_{2}\right)$; IR (neat, $\left.\mathrm{cm}^{-1}\right) 3453,3066,3037,2980,2935,2890,2360$, $1958,1882,1732,1496,1375,1263,1183,1062,918,851,757,699,650,584 ;{ }^{1} \mathrm{H}$ NMR $\left(\mathrm{CDCl}_{3}, 600 \mathrm{MHz}\right) \delta 7.49(\mathrm{~m}, 2 \mathrm{H}), 7.33(\mathrm{~m}, 3 \mathrm{H}), 5.58(\mathrm{~s}, 1 \mathrm{H}), 4.40$ (ddd, $J=7.8,6.0,2.4 \mathrm{~Hz}$, $1 \mathrm{H}), 4.14(\mathrm{dq}, J=7.2,2.4 \mathrm{~Hz}, 2 \mathrm{H}), 4.10(\mathrm{dq},, J=6.6,2.4 \mathrm{~Hz}, 1 \mathrm{H}), 2.69(\mathrm{dd},, J=15.6,7.8$ $\mathrm{Hz}, 1 \mathrm{H}), 2.48(\mathrm{dd}, J=15.6,6.0 \mathrm{~Hz}, 1 \mathrm{H}), 1.54(\mathrm{qdd},, J=7.2,2.4,2.4 \mathrm{~Hz}, 1 \mathrm{H}), 1.27(\mathrm{~d},, J=$ $6.6 \mathrm{~Hz}, 3 \mathrm{H}), 1.26(\mathrm{t}, J=7.2 \mathrm{~Hz}, 3 \mathrm{H}), 1.01(\mathrm{~d}, J=7.2 \mathrm{~Hz}, 3 \mathrm{H}) ;{ }^{13} \mathrm{C} \mathrm{NMR}\left(\mathrm{CDCl}_{3}, 150 \mathrm{MHz}\right)$ $\delta$ 170.9, 138.5, 128.6, 128.1 (2C), 126.1 (2C), 101.6, 77.2, 76.4, 60.4, 37.9, 35.4, 18.5, 14.1, 5.5; HRMS (ESI) calcd for $\left[\mathrm{C}_{16} \mathrm{H}_{22} \mathrm{O}_{4}+\mathrm{Na}\right]^{+}:$301.1410, Found: 301.1422 .

\section{Supplementary Material}

Refer to Web version on PubMed Central for supplementary material.

Acknowledgment

We are grateful to NIH (GM63150) and NSF (CHE-0415469) for the support of our research program and NSFEPSCoR (0314742) for a $600 \mathrm{MHz}$ NMR and an LTQ-FT Mass Spectrometer at WVU.

\section{References}

1. a Smith CM, O'Doherty GA. Org. Lett 2003;5:1959-1962. [PubMed: 12762696] b Garaas SD, Hunter TJ, O'Doherty GA. J. Org. Chem 2002;67:2682-2685. [PubMed: 11950317] c Hunter TJ, O'Doherty 
GA. Org. Lett 2001;3:2777-2780. [PubMed: 11506632] d Hunter TJ, O'Doherty GA. Org. Lett 2002;4:4447-4450. [PubMed: 12465909]

2. Hunter TJ, O'Doherty GA. Org. Lett 2001;3:1049-1052. [PubMed: 11277792]

3. Evans DA, Gauchet-Prunet JA. J. Org. Chem 1993;58:2446-2453.

4. For total synthesis of mycoticin A see: a Poss CS, Rychnovsky SD, Schreiber SL. J. Am. Chem. Soc 1993;115:3360-3361. For formal syntheses and approaches toward mycoticin A see: b Smith AB, Pitram SM. Org. Lett 1999;1:2001-2004. [PubMed: 10836057] c Dreher SD, Leighton JL. J. Am. Chem. Soc 2001;123:341-342. [PubMed: 11456525] For excellent background on oxo polyene macrolide antibiotics see: Rychnovsky SD. Chem. Rev 1995;95:2021-2040. and OmuraSMacrolide Antibiotics: Chemistry, Biology and Practice2002Academic PressNew York2nd Edition

5. For recent approaches to $\gamma$-methyl- $\delta$-hydroxyenoates, see: ref. ${ }^{4}$. and Chen Y-H, McDonald FE. J. Am. Chem. Soc 2006;128:4568-4569. [PubMed: 16594682] b Jain NF, Takenaka N, Panek JS. J. Am. Chem. Soc 1996;118:12475-12476. c Bluet G, Bazan-Tejeda B, Campagne J-M. Org. Lett 2001;3:3807-3810. [PubMed: 11700144] d Shirokawa, S.-i.; Kamiyama, M.; Nakamura, T.; Okada, M.; Nakazaki, A.; Hosokawa, S.; Kobayashi, S. J. Am. Chem. Soc 2004;126:13604-13605. [PubMed: 15493903] e Roush WR, Palkowitz AD, Ando K. J. Am. Chem. Soc 1990;112:6348-6359. f Rychnovsky SD, Hoye RC. J. Am. Chem. Soc 1994;116:1753-1765. g Schlessinger RH, Li Y-J. J. Am. Chem. Soc 1996;118:3301-3302. h Enders D, Voith M. Synlett 2002:29-32. i Denmark SE, Fujimori S. J. Am. Chem. Soc 2005;127:8971-8973. [PubMed: 15969572]

6. Tsuji has demonstrated a diastereoselective Pd-catalyzed reduction of trisubstituted vinyl epoxides, see: Oshima M, Yamazaki H, Shimizu I, Nisar M, Tsuji J. J. Am. Chem. Soc 1989;111:6280-6287.

7. a Tholander J, Carreira EM. Helv. Chim. Acta 2001;84:613-622. b Smith AB, Walsh SP, Frohn M, Duffey MO. Org. Lett 2005;7:139-142. [PubMed: 15624997]

8. Lower yields for the TBS series were due to minor loss of the TBS group in the carbonate-forming step.

9. Carreira has prepared $\mathbf{1 9}$ by a three step sequence from $\mathbf{6 d}$, see: ref. $7 \mathrm{a}$.

10. The regioselectivity of the asymmetric dihydroxylation of di- and trienoates has been studied by Sharpless and our group, see: a Berker H, Soler MA, Sharpless KB. Tetrahedron 1995;51:1345. and b Zhang Y, O'Doherty GA. Tetrahedron 2005;61:6337-6351.

11. This constitutes a reduction in the ratio of $\mathrm{Pd}$ vs phosphine to our optimized conditions for the desmethyl substrates, but is in accordance with Tsuji's vinyl epoxide reduction, albeit with significantly lower catalyst loadings, see: ref. ${ }^{6}$.

12. The ${ }^{1} \mathrm{H}$ NMR spectrum showed a qdd multiplicity $\left(J_{H a H b}=J_{H b H c}=2.4 \mathrm{~Hz}\right)$ for the proton at $C-4$, indicating the all syn-stereochemistry of both $\mathbf{1 4}$ and $\mathbf{1 5}$, see supporting information.

13. It is noteworthy that the dihydroxylation of $\mathbf{1 6}$ appears to proceed with the opposite facial selectivity to the analogous $(E, E)$-diene but this result is consistent with the Sharpless mnemonic, see: Kolb HC, VanNieuwenhze MS, Sharpless KB. Chem. Rev 1994;94:2483-2547.

14. Shi Y. Acc. Chem. Res 2004;37:488-496. [PubMed: 15311947]

15. In general, the all-equatorial diastereomer $\mathbf{2 1}$ forms with even greater stereocontrol than $\mathbf{1 5}$.

16. Presented in this Experimental Section are the general experimental procedures and spectral data for all new compounds. Complete experimental procedures and spectral data for all compounds are presented in the Supporting Information. 


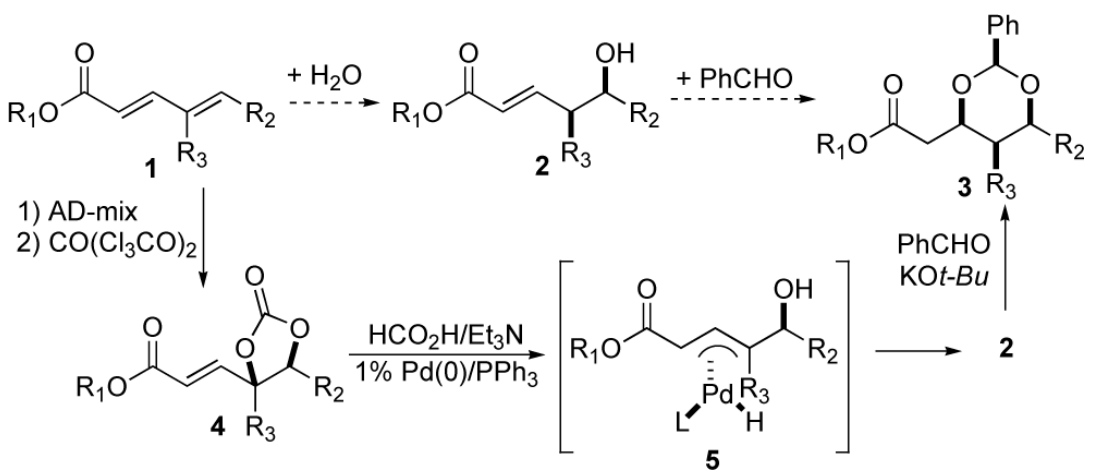

Scheme 1.

Asymmetric iterative hydration of C4-methyl dienoates 
<smiles>[R]OC(=O)/C=C/[C@@H](C)[C@H]([18OH])C(C)C</smiles>

27

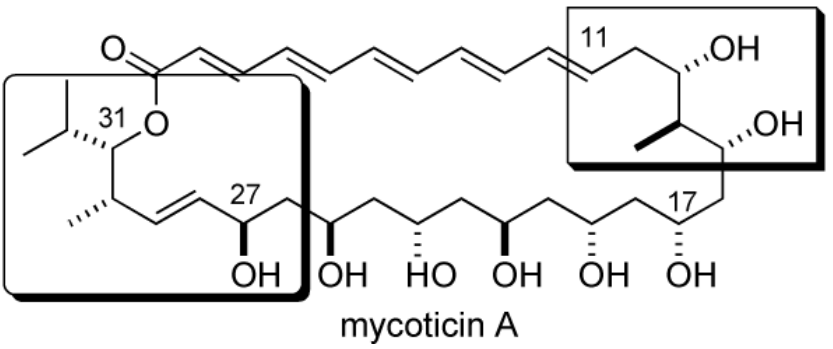<smiles>[R]OCC[C@H]1O[C@@H](c2ccccc2)O[C@H](CC=[Pt])C1C</smiles>

17

Figure 1.

Asymmetric hydration approach to mycoticin A 
<smiles>[R]OCC/C=C(C)/C=C/C(=O)OCC</smiles>

6a: $R=T B S$

6b: $R=B n$

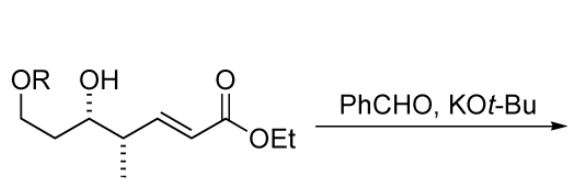

8a: $R=$ TBS; $68 \%$ yield

8b: $R=B n ; 84 \%$ yield

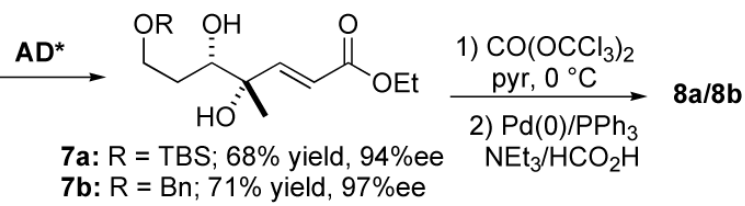

$\mathrm{Ph}$

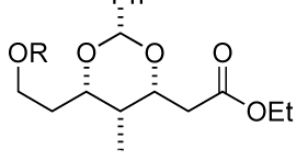

9a: $R=$ TBS; $58 \%$ yield, $d r=88: 12$ $9 \mathrm{~b}: \mathrm{R}=\mathrm{Bn} ; 69 \%$ yield, $\mathrm{dr}=89: 11$

$\mathrm{AD}^{*}=1 \% \mathrm{OsO}_{4}, 5 \%(\mathrm{DHQ})_{2} \mathrm{PHAL} 3 \mathrm{eq} \mathrm{K} \mathrm{K}_{3} \mathrm{Fe}(\mathrm{CN})_{6}, 3 \mathrm{eq}$

$\mathrm{K}_{2} \mathrm{CO}_{3}$ 3eq $\mathrm{MeSO}_{2} \mathrm{NH}_{2}, 0.2 \mathrm{M} t-\mathrm{BuOH} / \mathrm{H}_{2} \mathrm{O}(1: 1), 0^{\circ} \mathrm{C}-\mathrm{RT}$

Scheme 2.

Asymmetric double hydration of (E,E)-dienoate 

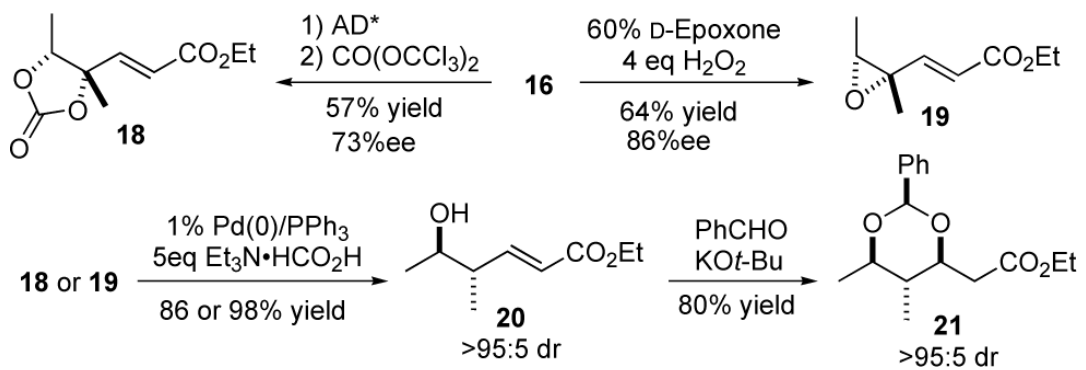

Scheme 3.

Shi epoxidation of (E,Z)-dienoates 


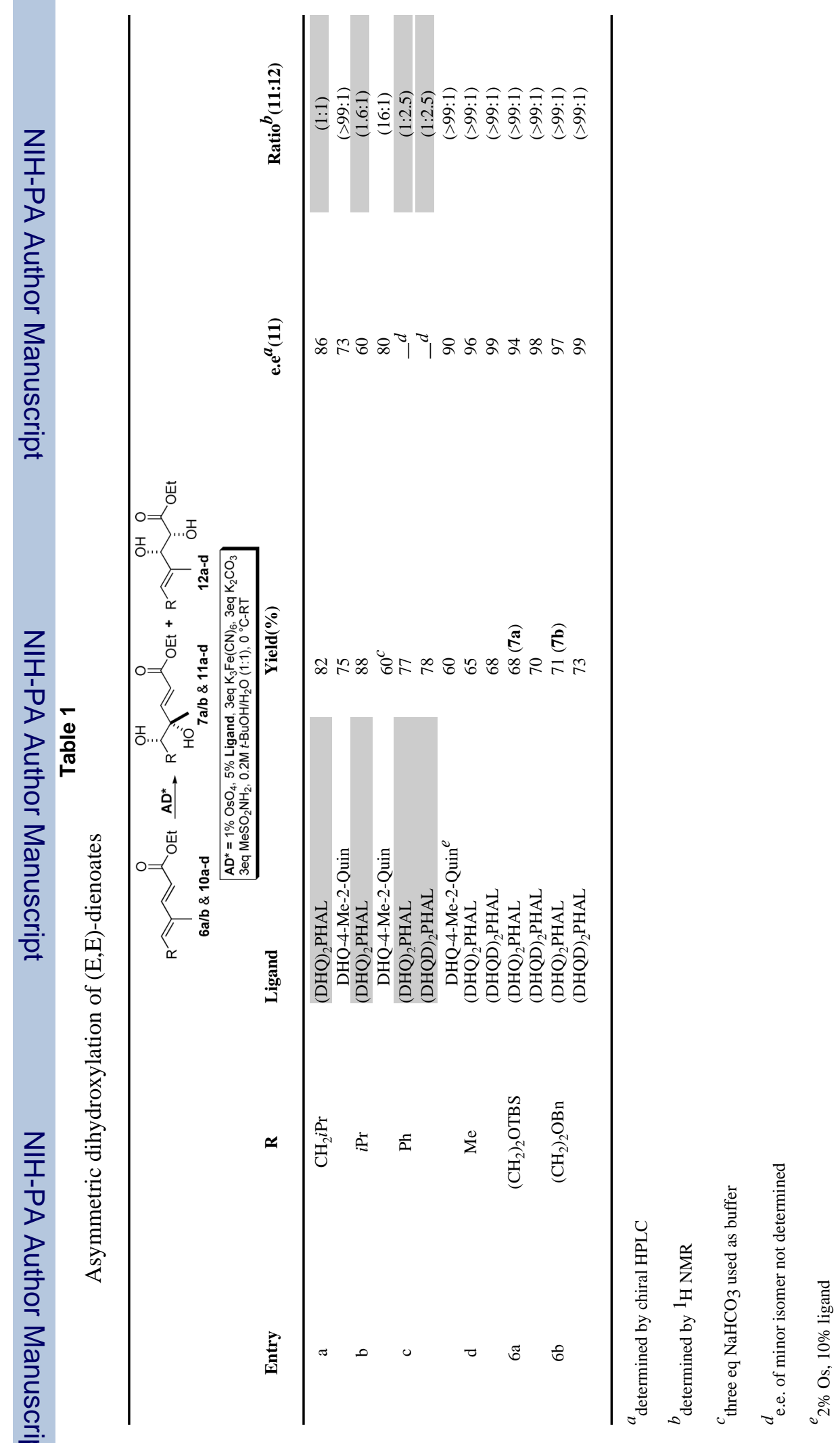




\section{Table 2}

Diastereoselective carbonate reduction

\begin{tabular}{|c|c|c|c|c|}
\hline \multirow[b]{3}{*}{ Entry } & \multirow[b]{3}{*}{$\mathbf{R}$} & R & Ollo \& 14a-e & \multirow[b]{3}{*}{$\mathrm{dr}^{a}$} \\
\hline & & \multicolumn{2}{|c|}{\begin{tabular}{|l|}
$\mathrm{Pd}(0)=1 \% \mathrm{Pd}_{2}(\mathrm{dba})_{3} \cdot \mathrm{CHCl}_{3}, 1 \% \mathrm{PPh}_{3}, 5 \mathrm{eq}$ \\
$\mathrm{NEt}_{3}, 5$ eq $\mathrm{H}_{2} \mathrm{CO}_{2}, 0.2 \mathrm{M} \mathrm{THF}$, reflux $10-40 \mathrm{~min}$
\end{tabular}} & \\
\hline & & Yield\% (13) & Yield\% (14) & \\
\hline $\mathrm{a}$ & $\mathrm{CH}_{2} i \mathrm{Pr}$ & 90 & 96 & $>95: 5$ \\
\hline $\mathrm{b}$ & $i \operatorname{Pr}$ & 84 & 98 & $>95: 5$ \\
\hline $\mathrm{c}$ & $\mathrm{Ph}$ & 91 & 98 & $>95: 5$ \\
\hline d & $\mathrm{Me}$ & 90 & 98 & $>95: 5$ \\
\hline $\mathrm{e}$ & $\operatorname{Me}(\text { ent }-1)^{b}$ & 90 & 98 & $>95: 5$ \\
\hline $7 a$ & $\left(\mathrm{CH}_{2}\right)_{2} \mathrm{OTBS}$ & $71(\mathbf{1 3 f})$ & $95(\mathbf{8 a})$ & $>95: 5$ \\
\hline $7 \mathrm{~b}$ & $\left(\mathrm{CH}_{2}\right)_{2} \mathrm{OBn}$ & 88 (13g) & $96(\mathbf{8 b})$ & $>95: 5$ \\
\hline
\end{tabular}

\footnotetext{
$a_{\text {determined by }}{ }^{1}$ H NMR

$b_{\text {dienoate subjected to (DHQD) } 2 \text { PHAL }}$
} 
Table 3

Diastereoselective hydration of $\delta$-hydroxyenoates

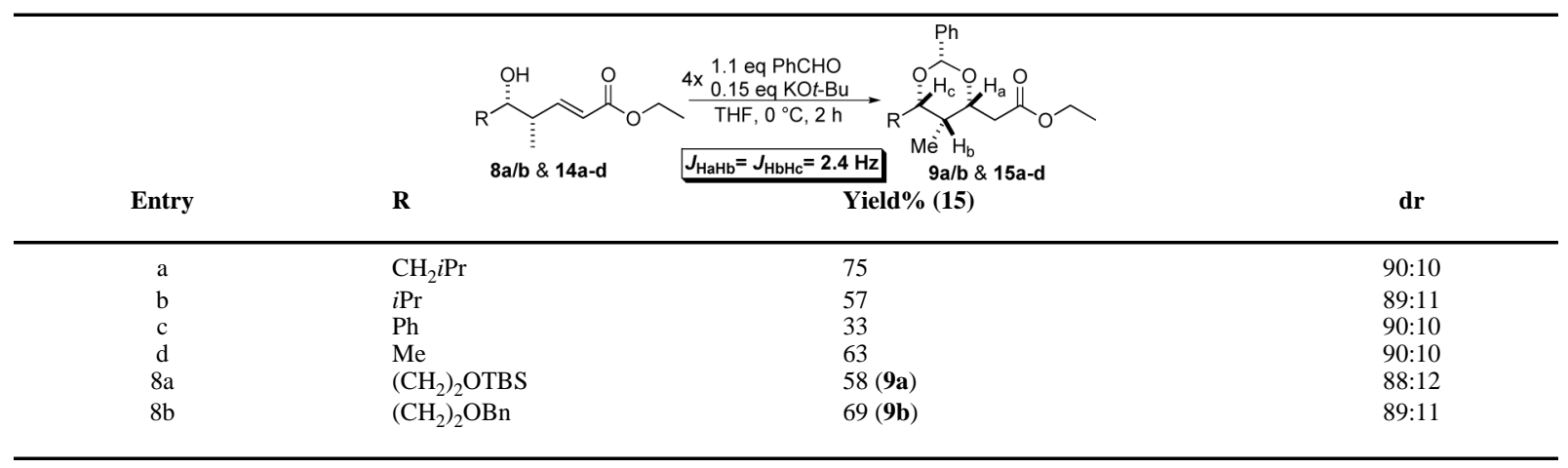




\section{Table 4}

Asymmetric dihydroxylation of (E,Z)-dienoates

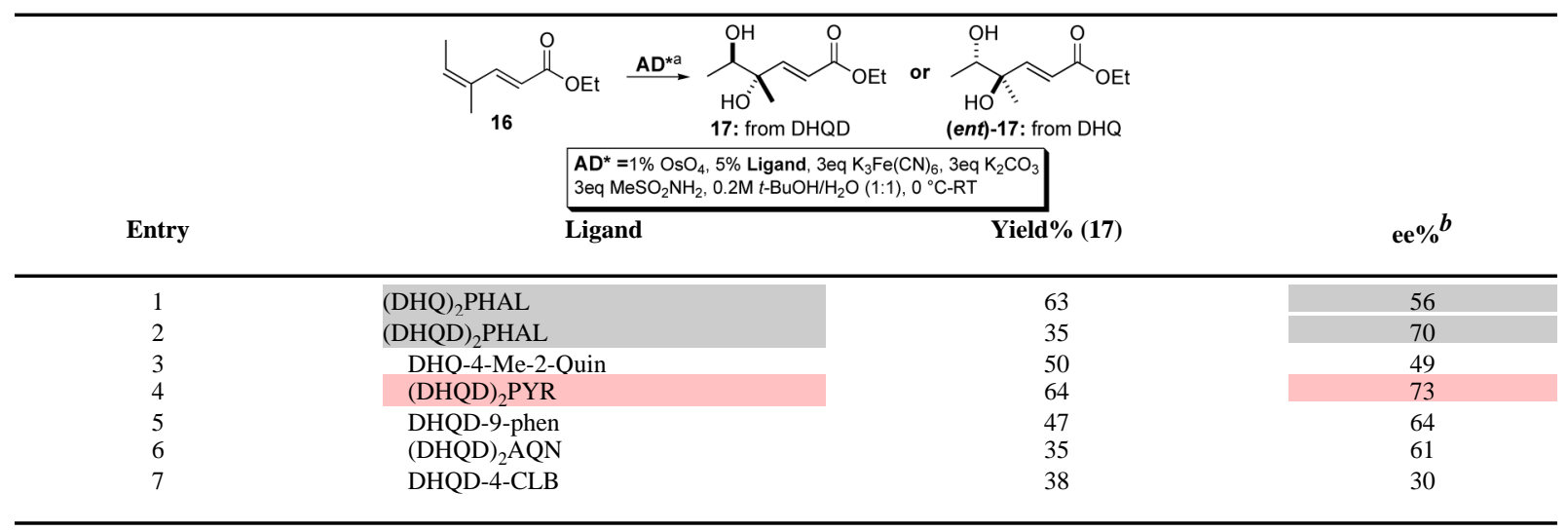

a) $\mathbf{1 7}$ is major from DHQD ligands whereas (ent)-17 is major from DHQ ligands.

b ee\% were determined by chiral HPLC 